\title{
Let Us Not Forget Cardiovascular Diseases During COVID-19 Pandemic: the Role of Cardiac Prevention and Rehabilitation
}

\author{
Leonarda Galiuto ${ }^{1}$ (D) $\cdot$ Filippo Crea ${ }^{1}$
}

Accepted: 1 July 2020 / Published online: 10 July 2020

(C) Springer Nature Switzerland AG 2020

\begin{abstract}
During novel coronavirus disease (COVID-19) pandemic, major focus of health service is on mitigating the spread of infection and treating the acute severe respiratory syndrome of affected patients. However, from available initial data, it has been shown that cardiovascular and metabolic diseases are responsible for a worse clinical outcome of COVID-19 patients and, on the other hand, myocardial damage might occur as a consequence of infection. Therefore, we propose not to forget the heart during pandemic and to focus on cardiac care in at least three phases: prevention, acute phase, and rehabilitation. We report rationale, scientific evidence, and clinical model for the proposed three-phase program.
\end{abstract}

Keywords Exercise $\cdot$ Cardiac rehabilitation · Prevention · COVID-19 · SARS-CoV2 - Pandemic

\section{Introduction}

Since 2019, the World Health Organization (WHO) called non-communicable disease as major health alert, and exercise-based cardiac prevention and rehabilitation programs were valorized as a precious tool to improve population health [1].

Although during severe acute respiratory syndrome due to a novel coronavirus (SARS-CoV2) [2], global health systems need to focus on treatment and mitigation of COVID-19, the overall impact of non-communicable disease even in this setting needs to be taken into account. Studies and a metaanalysis made clear that cardiovascular metabolic comorbidities made patients more susceptible to suffer COVID-19 and exacerbated the infection [3]. On the other hand, COVID-19 can produce or aggravate heart damage. The reasons for this bidirectional link remain to be investigated; however, we propose to keep the focus on the heart of COVID-19 patients in the prevention, treatment, and rehabilitation phases.

This article is part of the Topical Collection on Covid-19

Leonarda Galiuto

leonarda.galiuto@unicatt.it

1 Department of Cardiovascular Sciences, Fondazione Policlinico Universitario A. Gemelli-IRCCS, Catholic University of the Sacred Heart, Rome, Italy

\section{Phase 1: Prevention and Treatment of Risk Factors and Heart Disease}

In different series of COVID-19 patients, the most distinctive comorbidities were hypertension, cardiac and cerebrovascular disease, and diabetes, with an incidence of $17.1 \%, 16.4 \%$, and $9.7 \%$, respectively [3]. The incidence of such pathologic states was much higher in severe COVID-19 cases, being twofold, threefold, and twofold, respectively [3]. Thus, a cardiovascular metabolic disease not only predisposes to COVID-19 infection but also leads to a more severe clinical course.

At this time, given the very recent outbreak of the pandemic, and despite having strong correlation data, yet a causation cannot be automatically implied; thus, conceivable explanations for this association between comorbid states and COVID-19 can only be postulated. Since SARS-CoV2 virus infection is triggered by binding of spike protein of the virus to ACE2, which is not only highly expressed in lungs but also involved in the development of hypertension and diabetes mellitus, there is a possible link between COVID-19 and these two cardiovascular risk factors [4]. Furthermore, preliminary data found a significant higher ACE2 gene expression in smokers as compared to non-smokers, thus suggesting a possible susceptibility of smokers to SARS-CoV2. Thus, not only these patients should be considered susceptible to COVID-19 but also smoking cessation along with effective prevention and treatment of hypertension and diabetes appear to be necessary measures even during pandemic. 
After the first phase of viral infection, inflammatory response with cytokine release aggravates lung and multiorgan failure. Thus, it is conceivable that a healthy immune system and a non-inflammatory state, although not effective in preventing virus infection and replication, might be active in mitigating the inflammatory reaction of the SARS-CoV2 infection, thus possibly reducing additional systemic damage. Immunosenescence and inflammaging, determining increased susceptibility to infections, and reactivation of latent viruses might play a pivotal role in the higher prevalence of COVID19 with more complicated clinical course in aging population [5].

Exercise-based cardiac rehabilitation is the most effective preventive program to reduce the burden of cardiovascular risk factors on patients' health [1] and, along with optimal pharmacological treatment, need to be proposed in order to prevent aggressive effects of eventual virus exposure [5]. Moderate constant exercise should be encouraged and never stopped during the pandemic, since it not only exhibits wellknown beneficial effects on cardiovascular system but it is also able to support and increase immune health. Since sedentary habits, decreased muscle mass and poor nutritional status facilitate immunosenescence and inflammaging, promotion of physical activity and dietary intervention might lead to immunoprotection, with possible beneficial effects on secondary damage by COVID-19 [5]. Current recommendations for immune support also include stress management with yoga and meditation and a correct nutrition based on $>50 \%$ of energy coming from carbohydrate, adequate amount of protein and micronutrients, and a variety of fruit and vegetables every day [6].

\section{Phase 2: Treatment of Heart Disease During COVID-19}

Although clinical manifestation of COVID-19 is mainly respiratory, some patients have severe cardiovascular damage, as demonstrated by increased cardiac troponin levels, and reported cases of myocarditis and heart failure [3]. According to initial data, at least $8 \%$ of patients with COVID-19 suffered acute cardiac injury [3]. Although generally present at low levels, troponin elevation among patients hospitalized with COVID-19 is more often present in patients with cardiovascular disease and is associated with higher risk of mortality [7]. While myocardial injury has a significant association with fatal outcome of COVID-19, due to cardiac dysfunction and arrhythmias, the prognosis of patients with underlying cardiovascular disease but without myocardial injury were relatively favorable [8].

As compared with patients without cardiac injury, patients with cardiac injury were older; had more comorbidities; had higher leukocyte counts and levels of C-reactive protein,

\section{PREVENTION}
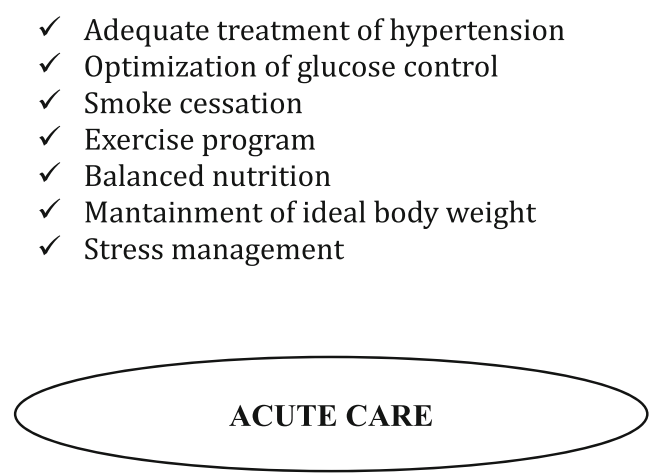

Evaluation of left ventricular function

$>$ Check for myocardial damage

$>$ Consider possible microvascular damage

$>$ Optimize oxygenation

$>$ Focus on management of previous cardiovascular disease

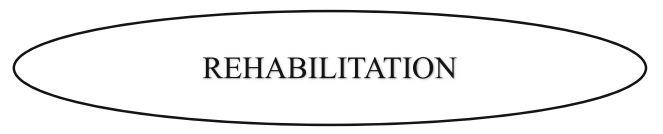

$\checkmark$ Assessment of possible dysregulation of lipid metabolism

$\checkmark$ Optimization of glucose control

$\checkmark$ Evaluation of post-acute left ventricular function

$\checkmark$ Assessment of cardio-pulmonary function during stress

$\checkmark$ Design personalized exercise programs

Fig. 1 Three-phase program of heart protection during COVID-19. We propose a three-phase program of heart care during COVID-19 pandemic. Based on current evidence, cardiovascular diseases should be prevented, treated in the acute phase, and proposed for a rehabilitation program

procalcitonin, creatinine kinase-myocardial band, myohemoglobin, high-sensitivity troponin I, N-terminal proB-type natriuretic peptide, aspartate aminotransferase, and creatinine; and had a higher proportion of multiple mottling and ground-glass opacity in radiographic findings, required noninvasive or invasive mechanical ventilation. Complications were more common in patients with cardiac injury than those without cardiac injury and included acute respiratory distress syndrome, acute kidney injury, electrolyte disturbances, hypoproteinemia, and coagulation disorders. Patients with cardiac injury had higher mortality than those without cardiac injury [9].

Therefore, it is reasonable to triage patients with COVID19 according to the presence of underlying CVD and evidence of myocardial injury for prioritized treatment and even more aggressive strategies. 
It is conceivable that heart damage in the acute phase of infection might be due to direct virus action on ACE2-expressing myocardial cells, or as a result of global inflammatory response and immune system disorders during disease progression. Hypoxemia may be also an important reason of cardiac injury. Preliminary, unpublished and unconfirmed autoptic data report the presence of systemic microvascular inflammation, which might also affect the heart. Recent data show the presence of SARS-CoV2 elements within endothelial cells and an accumulation of inflammatory cells, with evidence of endothelial and inflammatory cell death. COVID-19-endotheliitis could explain the systemic impaired microcirculatory function in different vascular beds and their clinical sequelae in patients with COVID-19 [10]. This data could be particularly relevant for vulnerable patients with preexisting endothelial dysfunction, which is associated with male sex, smoking, hypertension, diabetes, obesity, and established cardiovascular disease, all of which are associated with adverse outcomes in COVID-19 [10]. In addition, repeated floods of catecholamines due to anxiety and distress along with side effects of medications might also lead to myocardial damage. On the other hand, patients with underlining cardiovascular disease might have an increased risk of death, as a result of different pathophysiological mechanism and overall weakness and frailty [7-9]. Therefore, in this acute phase, specialized cardiovascular diagnosis and care should be proposed to be added to the already complex treatment of COVID-19 patients (Fig. 1).

\section{Phase 3: Rehabilitation After COVID-19}

Long-term follow-up of patients who recovered from SARS demonstrated that $68 \%$ has hyperlipidemia, $44 \%$ had cardiovascular disease, and 60\% had diabetes [11]. Lipid and glucose metabolism was demonstrated dysregulated by SARS$\mathrm{CoV}$ infection and chronic cardiovascular damage had been postulated. Therefore, not only cardiovascular protection should be given during COVID-19 infection, but also adequate cardiac rehabilitation programs should be proposed to survivors of the disease.

Given possible, although minor, myocardial damage in COVID-19 survivors, cardiac evaluation of all patients with previously known or suspected cardiovascular disease is desirable. Furthermore, in order to prescribe personalized exercise program to optimize secondary prevention, a cardiovascular and respiratory functional evaluation should be required possibly throughout a cardiopulmonary stress test.

\section{Conclusions}

The current COVID-19 pandemic fits to the 2008 WHO definition of health as "the ability to adapt and self-manage, in light of the physical, emotional and social challenges of life." In this challenge, while aggressively treating and mitigating the spread of COVID-19, health systems need not to lose the focus on the burden of non-communicable disease in order to limit the overall impact of infectious disease. Exercise-based cardiac prevention and rehabilitation models are likely reduce infection risk, might limit cardiovascular damage during the acute phase of infection and provide optimal recovery and long-term health benefits after the infection.

\section{Disclosure Statement}

This paper is not under consideration elsewhere; none of the paper's content has been previously published, and all authors have read and approved the manuscript. Both authors have contributed significantly to the submitted paper.

\section{Compliance with Ethical Standards}

Conflict of Interest The authors declare that they have no conflict of interest.

\section{References}

1. Anderson L, Oldridge N, Thompson DR, Zwisler AD, Rees K, Martin N, et al. Exercise-based cardiac rehabilitation for coronary heart disease cochrane systematic review and meta-analysis. J Am Coll Cardiol. 2016;67(1). https://doi.org/10.1016/j.jacc.2015.10. 044.

2. Wu Z, McGoogan JM. Characteristics of and important lessons from the coronavirus disease 2019 (COVID-19) outbreak in China. JAMA. 2020;323:1239. https://doi.org/10.1001/jama.2020. 264.

3. Li B, Yang J, Zhao F, Zhi L, Wang X, Liu L, et al. Prevalence and impact of cardiovascular metabolic diseases on COVID-19 in China. Clin Res Cardiol. 2020;109:531-8. https://doi.org/10. 1007/s00392-020-01626-9.

4. Zheng Y-Y, Ma Y-T, Xie X. COVID-19 and the cardiovascular system. Nature Reviews Cardiology. 2020;17:259-260.

5. Chen P, Mao L, Nassis GP, Harmer P, Ainsworth BE, Li F. Coronavirus disease (COVID-19): the need to maintain regular physical activity while taking precautions. J Sport Health Sci. 2020;9:103-4.

6. Davison G, Kehaya C, Jones AW. Nutritional and Physical activity interventions to improve immunity. Am J Lifestyle Med. 2014. 2016 May-Jun;10(3):152-69. https://doi.org/10.1177/ 1559827614557773.

7. Lala A, Johnson KW, Januzzi J, Russak AJ, Paranjpe I, Richter F, et al. Prevalence and impact of myocardial injury in patients hospitalized with COVID-19 infection. J Am Coll Cardiol. 2020. https:// doi.org/10.1016/j.jacc.2020.06.007.

8. Guo T, Fan Y, Chen M, Wu X, Zhang L, He T, et al. Cardiovascular implications of fatal outcomes of patients with coronavirus disease 
2019 (COVID-19). JAMA Cardiol. 2020. https://doi.org/10.1001/ jamacardio.2020.101.

9. Shi S, Qin M, Shen B, Cai Y, Liu T, Yang F, et al. Association of cardiac injury with mortality in hospitalized patients with COVID19 in Wuhan, China. JAMA Cardiol. 2020. https://doi.org/10.1001/ jamacardio.2020.0950.

10. Varga Z, Flammer AJ, Steiger P, Haberecker M, Andermatt R, Zinkernagel AS, et al. Endothelial cell infection and endotheliitis in COVID-19. Lancet. 2020;395(10234):1417-8. https://doi.org/ 10.1016/S0140-6736(20)30937-5 Published online 2020 Apr 21.
11. Wu Q, Zhou L, Sun X, Yan Z, Hu C, Wu J, et al. Altered lipid metabolism in recovered SARS patients twelve years after infection. Sci Rep. 2017;7:9110. https://doi.org/10.1038/s41598-01709536-z.

Publisher's Note Springer Nature remains neutral with regard to jurisdictional claims in published maps and institutional affiliations. 